\title{
MICROFLUIDIC FABRICATION OF MONODISPERSE MICROCAPSULES FOR SELF HEALING MATERIAL
}

\author{
R.J. Lemmens* and D.D. Meng
}

Mechanical Engineering - Engineering Mechanics Department

Michigan Technological University, Houghton, Michigan, USA

\begin{abstract}
We report microfluidic fabrication of microcapsules for use in self-healing composites which results in dramatic reduction of the coefficient of variation $(\mathrm{CV})$ of the microcapsule size from $\sim 40$ to less than 4, as compared to traditional batch emulsification methods. With this fabrication approach the mean microcapsule size can also be accurately controlled through variation of process parameters such as fluid flow rate. The surface morphology, i.e. roughness of the capsule shell, and shell thickness can also be tuned by utilizing fluid shear and controlling temperature.
\end{abstract}

\section{INTRODUCTION}

A self healing material is typically a composite, in many cases polymer, consisting of a bulk matrix with embedded healing agent. In a generic self healing model damage to the composite causes release of healing agent into the crack volume and polymerization of the healing agent results in reestablishment of mechanical properties [1]. Healing agent incorporation schemes include capsules, capillaries, fugitive ink scaffold writing as well as others. Possible intrinsic or extrinsic healing triggers include the addition of heat, pressure, moisture or the presence of catalyst or hardening agent [2]. Taking into account material and fabrication costs, ease of composite fabrication, and production scalability, the use of microcapsules as the means of healing agent incorporation is favored. As a constituent of the composite the properties of these microcapsules have a direct impact on the performance of self healing materials [3] and are therefore a crucial parameter in the engineering of such composites.

The size and surface roughness of the microcapsules can be critical for both the mechanical properties and the self-healing performance of the composite. Rule et al. showed that the degree of healing is directly related to the mass of healing agent delivered to the crack volume and that this mass is dependent only on the size and concentration of microcapsules incorporated into the composite [4] assuming a uniform distribution of microcapsules and a negligible shell thickness. An investigation by Brown et al. found that incorporation of microcapsules into an epoxy matrix resulted in significant fracture toughening [5]. The degree of toughening was seen to have a strong dependence on microcapsule size as well as adhesion between the epoxy matrix and microcapsule shells. These improvements; however, do not always trend in the same direction with respect to the various properties of the microcapsules, e.g. size and shell morphology, requiring an optimization of properties and performance. Such an optimization would be more easily implemented given a microcapsule fabrication technique having precise control over these microcapsule characteristics.

Currently, microcapsules for use in self-healing composites are produced using batch emulsification with an accompanying shell polymerization mechanism. A well established material system utilizes a poly-urea-formaldehyde (UF) capsule shell and dicyclopentadiene (DCPD) as the encapsulated healing agent [6]. In the production process an aqueous solution of the polymerization ingredients is placed in a beaker along with the healing agent which is immiscible in water. A mechanical stirrer is used to create an emulsion of the healing agent and a condensation polymerization is initiated to form the capsule shell.

Attempts have been made with this fabrication approach to control the surface roughness of the microcapsules [6]. Variation of polymerization time showed an increase in the thickness of a rough shell but smooth capsules either could not be obtained or the DCPD diffused out within a couple of days. Smooth capsules were obtained using a constant $\mathrm{pH}$ process but in this case yields were only $\sim 10 \%$. The thickness of the rough shell was also seen to be dependent on the agitation rate as a result of the interplay between the magnitude of the DCPD/water interfacial area and mass conservation of the polymer. The capability of controlling the surface roughness without sacrificing product yield and stability or alteration of the volume of healing agent contained in the microcapsules has not yet been realized.

Additionally, precise size control for the microcapsules is difficult with batch emulsification technology. The mean diameter of the microcapsules produced is directly related to the rotational rate of the mechanical stirrer used to create the emulsions. Increased agitation causes increase shear and therefore, with a given interfacial tension, smaller DCDP droplets on average. The shear forces however are not homogeneous throughout the emulsion bath and DCPD droplet formation is not localized resulting in a broad distribution of microcapsule size. The coefficient of variation (CV) of the size distribution of the as produced microcapsules typically ranges between 20 and 50 . A significant reduction in this distribution would require improved control over droplet formation.

Droplet microfluidics is well known for its reliable and repeatable droplet production. It has been proven to be an invaluable platform for digital fluidics applications, biomaterial encapsulation and handling, as well as producing functional microreactors for studies in reaction kinetics [7]. The unique scaling within microchannels allows for precise control over fluid mixing and dominance of viscous and interfacial forces permitting controlled droplet formation and manipulation. The functionality of the microfluidic platform is adaptable to changes in fluid properties allowing a broad range of materials to be used. This provides optimal conditions for particle and microcapsule fabrication [8]. Incorporation of such advantages into the fabrication process for microcapsules used in self healing material would allot significant improvements in process controllability.

In this work, we report an approach to combine droplet microfluidics, which can produce highly-monodisperse emulsions, with an established condensation polymerization technique for shell formation to produce monodisperse microcapsules for selfhealing material applications. Facile control over shell surface morphology is also demonstrated.

\section{EXPERIMENTAL}

\section{Microcapsules Fabrication}

All materials are used as purchased. Dicyclopentadiene, urea, formaldehyde, ammonium chloride, resorcinol and sodium hydroxide were obtained through Sigma-Aldrich. Ethylene- 


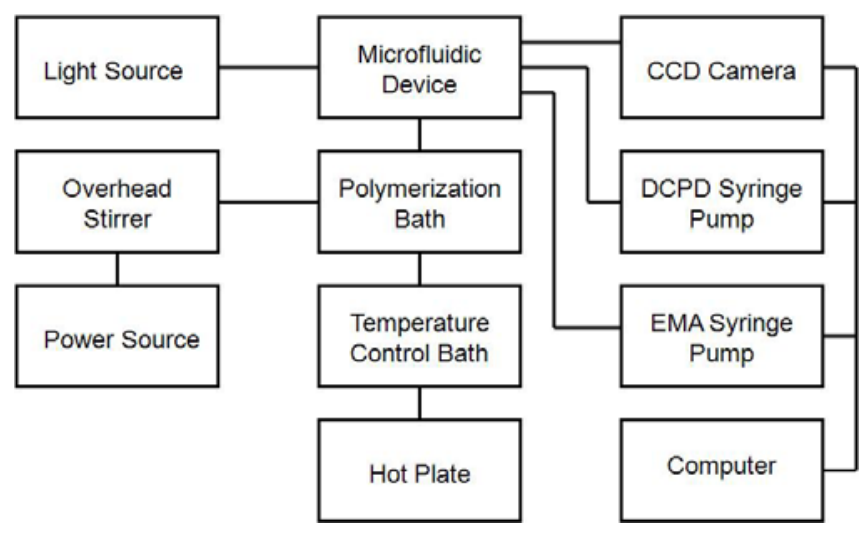

Figure 1: Schematic illustration of the microcapsules fabrication setup including microfluidic emulsification.

maleic-anhydride (EMA) was donated by Vertellus Specialties Inc. Sylgard 184 polydimethylsiloxane (PDMS) elastomer was purchased through Dow Chemicals.

The microcapsules production setup is shown in Figure 1. Two syringe pumps (Harvard Apparatus) are used to supply DCPD and a $2.5 \mathrm{wt} \%$ aqueous solution of EMA to the inlet channels of the PDMS microfluidic emulsifier. A computer is used to control the syringe pumps as well as collect video from a ccd camera (Moticam 2000) used to monitor the emulsification process within the device.

The PDMS device is produced using soft lithography techniques. The cross junction geometry of the microchannels acts as a flow focusing emulsifier [7]. The outlet channel dimensions at the cross junction are $60 \mu \mathrm{m}$ wide by $90 \mu \mathrm{m}$ deep for all emulsifications. Channel dimension are reduced to $60 \mu \mathrm{m} \times 70 \mu \mathrm{m}$ for one device to demonstrate the effects of channel size. The microchannels are oxygen plasma treated (Diener Electronics Femto System) to ensure proper surface wettability and sealed by bonding to a glass slide. An example of a monodisperse emulsification within this device is shown in Figure 2.

Formation of the microcapsule shell occurs in a bath into which the emulsion from the microfluidic device is collected. The chemistry and polymerization mechanism is similar to [6]. Urea (1g), ammonium chloride (100mg), and resorcinol $(100 \mathrm{mg})$ are desolved in $40 \mathrm{ml}$ of water and placed in an $80 \mathrm{ml}$ beaker along with $5 \mathrm{ml}$ of a $2.5 \mathrm{wt} \%$ aqueous solution of EMA. This bath is agitated with an overhead stirrer using a 1 inch, 3 blade impeller. Agitation is performed at a relatively low $200 \mathrm{rpms}$ so as to maintain droplet integrety.

Emulsification is performed with constant EMA and DCPD flow rates for a total of $100 \mathrm{~min}$. prior to shell formation. After collection of the emulsion the $\mathrm{pH}$ of the polymerization bath is increased from $\sim 2.6$ to 3.5 by dropwise addition of sodium hydroxide. Formaldehyde $(2.634 \mathrm{~g})$ is then added to the bath and the temperature is increased from room temperature to $55{ }^{\circ} \mathrm{C}$ in order to initiate polymerization. The temperature is maintained using a hotplate and water bath for $4 \mathrm{hrs}$. after which agitation is stopped, heat is removed, and the bath is strained through a coarse fritted filter. After washing with DI water the product is allowed to dry for $24 \mathrm{hrs}$. This process results in the collection of UF microcapsules containing DCPD in the form of a fine white powder.

For comparison microcapsules are also produced using batch emulsification. This procedure follows that outlined in [6]. Three samples of microcapsules were produced using different agitation rates, 600,650 and $750 \mathrm{rpm}$, in an attempt to produce

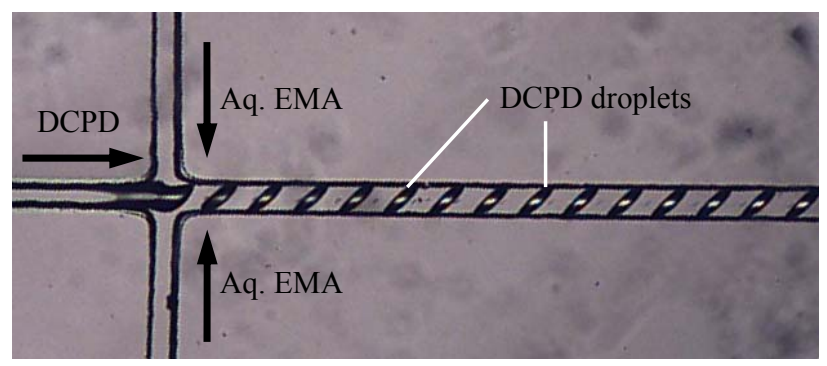

Figure 2: Emulsification of DCPD at the flow focusing cross junction of a microfluidic droplet generator. EMA and DCPD flow rates are $45 \mu \mathrm{L} / \mathrm{min}$ and $15 \mu \mathrm{L} / \mathrm{min}$ respectively.

microcapsules with a mean diameter similar to those produced using the microfluidic emulsification technique.

In order to demonstrate control of surface morphology, post polymerization processing were performed to rapidly quench the polymerization bath to room temperature. Spcificaly, after agitation of the polymerization bath is ceased, the contents of the bath are poured into $1 \mathrm{~L}$ of deionized water. Filtration, washing, and product drying then follow.

\section{Characterization}

Size analysis of the microcapsules is performed using an image analysis software, Image $J^{\circledR}$. Samples of the microcapsules are placed on a glass slide and optically imaged using a ccd camera and Motic Images Plus software. A minimum of 300 microcapsules for each sample are analyzed to find the mean diameter and standard deviation of the distribution.

The surface morphology of the microcapsules is observed by Field Emission Scanning Electron Microscopy. To view the cross section of the shell microcapsules are manually ruptured and the DCPD is allowed to evaporate prior to sample preparation. Microcapsules are also sputtered with $5 \mathrm{~nm}$ of titanium prior to observation.

\section{RESULTS}

\section{Control of Capsule Size}

In this study control over the size of microfluidicly produced microcapsules is demonstrated through variation of fluid flow rates and microchannel dimensions. The size distribution of these microcapsules is also compared against that of those produced using the standard microcapsule production method.

Four samples of DCPD-containing UF microcapsules prepared using the microfluidic fabrication approach described above are presented in Figure 3 as samples A-D. The DCPD flow rate for these four samples was held constant at $15 \mu \mathrm{L} / \mathrm{min}$. Samples A-C were prepared using a PDMS devices whose channel dimensions were $60 \mu \mathrm{m}$ wide and $90 \mu \mathrm{m}$ deep with EMA flow rates of 15,45 , and $75 \mu \mathrm{L} / \mathrm{min}$ respectively. The channel dimensions were altered to $60 \mu \mathrm{m}$ wide and $70 \mu \mathrm{m}$ deep for Sample D and an EMA flow rate of $75 \mu \mathrm{L} / \mathrm{min}$ was used. Sample E was prepared by traditional batch emulsification techniques as a comparison and utilized an agitation rate of $650 \mathrm{rpm}$. Two additional samples were also prepared using batch emulsification with agitation rates of 600 and $750 \mathrm{rpm}$ but images are not shown.

It is found that the samples produced using microfluidics (AD) are monodisperse (typically defined as $\mathrm{CV}<5$ ) while the $\mathrm{CV}$ of control sample (E) is more than eight times greater. Each sample's mean diameter, coefficient of variation (CV), and corresponding normal distributions are shown in Figure 4. The two additional 


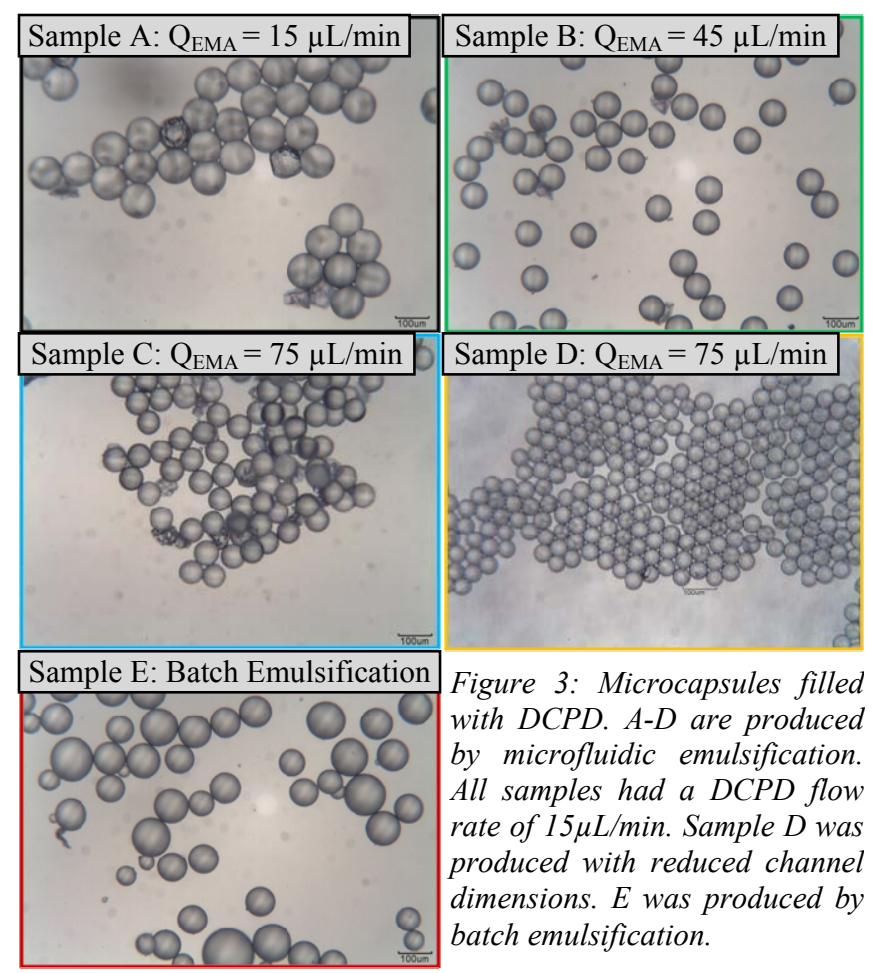

samples produced using batch emulsification were also highly polydisperse. The mean diameters were 88.5 and $65.6 \mu \mathrm{m}$ and the CVs were 25.8 and 45.1 for the 600 and $750 \mathrm{rpm}$ samples respectively.

It has been shown that the size of the microcapsules can be precisely controlled by altering the fluid flow rates and therefore the shear forces within the PDMS device. By tuning $Q_{\mathrm{EMA}}$, the mean capsule sizes of samples A-C have been controlled as 107.4, 80.7 , and $73.2 \mu \mathrm{m}$ respectively. Channel dimensions also play a similar role in control of capsules size as can be seen from Sample $\mathrm{D}$ which has a mean diameter of $53.4 \mu \mathrm{m}$.

The overall size distribution for each sample is attributed to the nature of droplet formation during the emulsification. With batch emulsification, shear forces are heterogeneous and droplet formation is delocalized. This is not the case for microfluidic emulsification. With constant flow rates of input fluids each droplet experiences similar shear and interfacial forces during formation resulting in a significant reduction in size distribution.

In comparing samples $\mathrm{A}$ and $\mathrm{D}$ to samples $\mathrm{B}$ and $\mathrm{C}$ it is seen that their CVs are slightly higher, by a factor of $\sim 2$. The increase seen for sample A can be explained by the coalescence of two or more droplets prior to shell formation. Such droplet coalescence was observed within the outlet channel of the PDMS device. It is conjectured that because the fluid flow rate within the outlet channel was low the tendency for contact between droplets and therefore merging was higher. A decrease of EMA flow rate to $10 \mu \mathrm{L} / \mathrm{min}$ was found to further increase the tendency of droplet coalescences to a point where monodispersity is lost.

The increase in CV seen for sample D can be explained by a transition into a different flow regime. The decrease in channel dimensions for this sample is accompanied by an increase in fluid velocity and therefore the Weber number of the flow increases. The transition from dripping to jetting in microchannels is strongly dependent on Weber number and the transition consists of a number of distinct regimes. In the initial stages of the transition multiple dripping occurs. In this regime the location of droplet

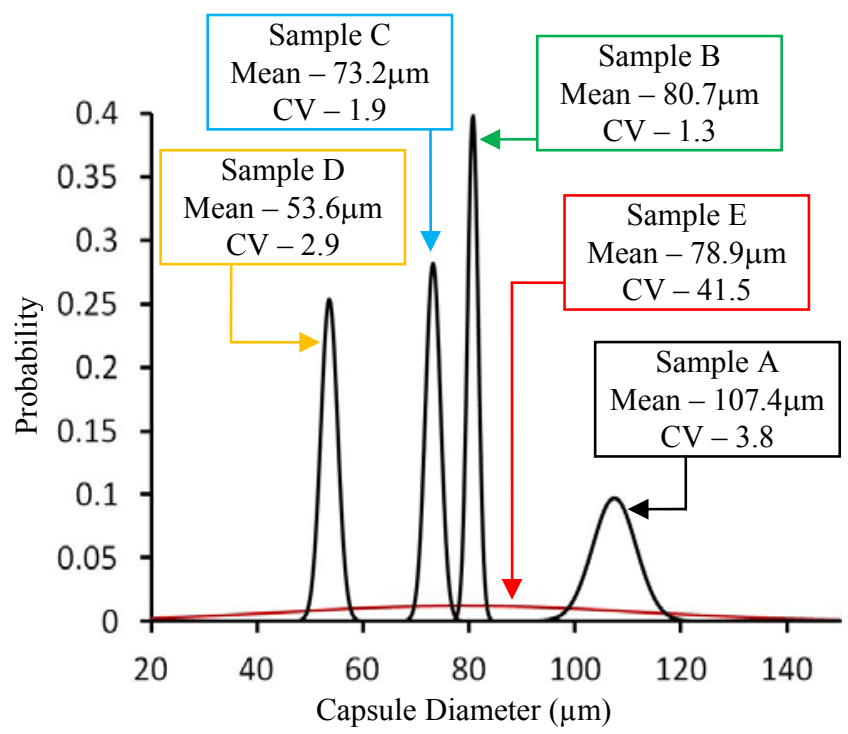

Figure 4: Comparison of the normal distributions of microcapsule diameter of samples produced using microfluidics, $A-D$, and that produced using current microcapsule fabrication techniques, $E$.

formation does not yet move into the outlet channel, as it does for jetting, however a droplet formation event does not produce a single droplet but rather multiple droplets which can vary in size [9].

Although samples A and D seem to have been produced with a broader size distribution the products still fall into the definition of being monodisperse. The observations made for these two sample reveals some of the guidelines which must be followed for this fabrication process. A window of operational flow rates will be specified for given channel dimensions where at the lower limit droplet coalescence is a risk and at the upper limit transition out of the dripping regime will occur. With that said use of a single device still provides the capability of producing microcapsule of various sizes and with fine tuned precision.

\section{Control of Capsule Surface Morphology}

Formation of the microcapsules shell results from a condensation polymerization of urea with formaldehyde which occurs in the continuous, i.e. aqueous, phase of the emulsion [6]. Shell formation begins at the interface between the DCPD droplets and the aqueous phase where in the early stages of polymerization low molecular weight UF is deposited. As the polymerization progresses further, the low molecular weight UF at the DCPD surface continues to crosslink forming a solid, continuous and smooth shell. Simultaneously, high molecular weight UF which is not soluble in water begins to precipitate out of solution in the form of nanoparticles. Deposition of these nanoparticles onto the smooth inner shell results in the formation of a rough outer shell. It was conjectured that during the polymerization and toward the end of the fabrication process a significant amount of UF nanoparticles form and remain in suspension in the continuous phase of the bath. The agitation from the mechanical stirrer keeps these particles as well as the microcapsules in constant motion. The relative motion between the particles causes hydrodynamic lubrication preventing intimate contact from being made. Removal of this agitation and the continued presence of the polymerization reaction permit deposition of the nanoparticles and cross linking to each other and the microcapsules surface. Development of the rough outer surface should therefore be controllable. 

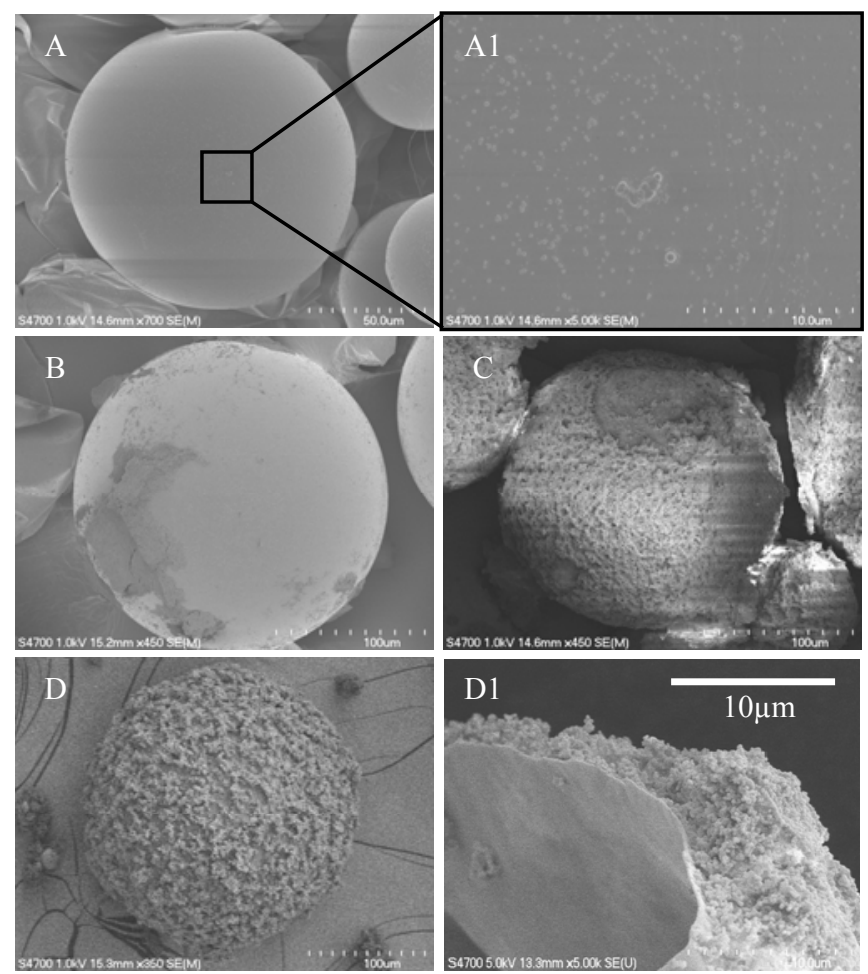

Figure 5: Evolution of microcapsule shell roughness with time elapsed after discontinuation of bath agitation until temperature quenching to room temperature (A) $40 s$ (B) 120 s (C) $200 s$ (D) 280 s (A1) Smooth capsule surface with minimal UF particle deposition (D1) Broken capsule from sample D showing smooth inner surface and representative thickness of rough outer surface.

Control of the microcapsules surface morphology is achieved by tuning the time elapsed between ceasing agitation of the polymerization bath and temperature quenching to minimize further polymerization. Four samples with various degrees of surface roughness are presented in Figure 5. The times used for the study were $40,120,200$, and 280 s for samples A-D respectively. The yields for all samples were between 80 and $90 \%$, where yield is measured as the ratio of the weight of the product capsules to the weight of the DCPD used in the emulsification. Losses are conjectured to be due to a combination of DCPD evaporation prior to shell formation and loss of product during filtration and collection.

Sample A was produced using the shortest time and possesses the smoothest surface. Figure 5A1 shows a magnified view of the surface where minimal UF particle deposition has occurred. The quality of these capsules is good in that they are mechanically stable, form a fine powder after drying, and DCPD does not leak or diffuse through the capsule shell. This quality has been found to remain for a minimum of 3 months with no signs of degradation. The effluent from the initial filtration of these microcapsules from the polymerization bath had a milky white color indicating that UF nanoparticles of high concentration still remain in suspension.

Samples B and C show that the surface roughness does not develop uniformly. This is conjectured to be due to UF particle agglomeration in suspension followed by deposition on the microcapsules surface.

Sample D was produced using the longest time and possesses a rough surface which is uniform over the whole shell and was seen to be consistent for other microcapsules from the same sample. Figure 5D1 shows the cross section of the shell of a microcapsule from this sample. A significant shell thickness has developed for these microcapsules although not uniform due to the textured nature of the surface. The effluent from the initial filtration of these microcapsules from the polymerization bath was clear indicating the absence of UF particles in suspension. Further increase in the time elapsed beyond that used in this sample did not show a significant change in surface morphology.

\section{CONCLUSION}

A microfluidic method of fabrication of microcapsules for use in self-healing material has been demonstrated. Fine tuning of the microcapsule diameter is performed through variation of fluid flow rates and selection of microchannel dimensions. The microcapsules produced are monodisperse representing a significant decrease by a factor of $\sim 10$ of the coefficient of variation when compared to those produced using traditional methods. The surface morphology of the microcapsules has also been controlled. Microcapsules with smooth and rough surfaces are produced with good quality and high yields.

\section{ACKNOWLEDGEMENT}

This material is based upon work supported by the National Science Foundation under Grant No. 0900582. Any opinions, findings, and conclusions or recommendations expressed in this material are those of the authors and do not necessarily reflect the views of the National Science Foundation.

Travel support has been generously provided by the Transducer Research Foundation.

The authors would also like to thank all members of the Multi-Scale Energy Systems Laboratory for their help and support.

\section{REFERENCES}

[1] S.R. White, et al., "Autonomic healing of polymer composites," Nature, vol. 409, pp. 794-797, 2001.

[2] B.J. Blaiszik, et al., "Self-Healing Polymers and Composites," Annual Review of Materials Research, vol. 40, pp. 179-211, 2010.

[3] M. Keller and N. Sottos, "Mechanical Properties of Microcapsules Used in a Self-Healing Polymer," Experimental Mechanics, vol. 46, pp. 725-733, 2006.

[4] J.D. Rule, et al., "Effect of microcapsule size on the performance of self-healing polymers," Polymer, vol. 48, pp. 3520-3529, 2007.

[5] E.N. Brown, et al., "Microcapsule induced toughening in a self-healing polymer composite," Journal of Materials Science, vol. 39, pp. 1703-1710, 2004.

[6] E.N. Brown, et al., "In situ poly(urea-formaldehyde) microencapsulation of dicyclopentadiene," Journal of Microencapsulation, vol. 20, pp. 719-730, 2003.

[7] S.-Y. Teh, et al., "Droplet microfluidics," Lab on a Chip, vol. 8, pp. 198-220, 2008.

[8] J.-T. Wang, et al., "Fabrication of Advanced Particles and Particle-Based Materials Assisted by Droplet-Based Microfluidics," Small, vol. 7, pp. 1728-1754, 2011.

[9] A.R. Abate, et al., "Impact of inlet channel geometry on microfluidic drop formation," Physical Review E, vol. 80, p. $026310,2009$.

\section{CONTACT}

* R.J. Lemmens, tel: 1-920-639-4028; rjlemmen@mtu.edu 